\title{
Impactação de dentes permanentes associado a odontoma composto em paciente infantil: relato de caso
}

Impact of permanent teeth associated with compound odontoma in a child: a clinical case Impacto de los dientes permanentes asociados con el odontoma compuesto en un niño: reporte de caso \author{
Matheus dos Santos FERNANDEZ ${ }^{1}$ \\ Laís Almeida SANTOS ${ }^{2}$ \\ Rayza Gabrielle Santos BARRETO \\ Cristiane Costa da Cunha OLIVEIRA ${ }^{3}$ \\ Vanessa dos Santos VIANA ${ }^{3}$
}

${ }^{1}$ Curso de Graduação em Odontologia, Faculdade de Odontologia, UFPel Univ. Federal de Pelotas, 96015-700 Pelotas - RS, Brasil ${ }^{2}$ Curso de Graduação em Odontologia, UNIT Univ. Tiradentes, 49010-390 Aracaju - SE, Brasil

${ }^{3}$ Professora do Curso de Graduação em Odontologia, UNIT Univ. Tiradentes, 49010-390 Aracaju - SE, Brasil

\section{Resumo}

Os odontomas são o tipo mais comum dos tumores odontogênicos benignos. Clinicamente apresentam-se assintomáticos e de crescimento lento, sendo divididos de acordo com as características histomorfológicas em (a) odontomas compostos, contendo tecidos dentários normais e formados por múltiplas estruturas calcificadas, algumas assemelhando-se a mini-dentes; e (b) odontomas complexos, quando presentes como massas irregulares contendo diferentes tecidos dentários. Normalmente estão associados ao atraso no irrompimento dentário. Um menino de 8 anos compareceu a Clínica Odontológica Universitaria com dúvidas sobre $o$ atraso na esfoliação dos dentes permanentes. Uma radiografia periapical evidenciou a presença de um odontoma composto na região dos incisivos superiores do lado esquerdo, constituindo um obstáculo à erupção permanente dos dentes. Sob anestesia local, o acesso ao tumor foi obtido via intra oral e sua enucleação cirúrgica foi realizada. A análise histopatológica confirmou o diagnóstico de odontoma composto. Uma revisão de literatura foi realizada para uma discussão mais profunda da temática.

Descritores: Tumores Odontogênicos; Odontoma; Erupção Dentária; Diagnóstico.

\section{Abstract}

Odontomas are the most common type of benign odontogenic tumors. Clinically, they are asymptomatic and slow-growing, being divided according to histomorphological characteristics into (a) compound odontomas, containing normal dental tissues and formed by multiple calcified structures, some resembling mini-teeth; and (b) complex odontomas, when present as irregular masses containing different dental tissues. They are usually associated with delayed tooth eruption. A 8-year-old boy attended the Clínica Odontológica Universitaria with doubts about the delay in exfoliating permanent teeth. A periapical radiography showed the presence of a compound odontoma in the region of the upper incisors on the left side, constituting an obstacle to the permanent eruption of the teeth. Under local anesthesia, access to the tumor was obtained via intraoral and its surgical enucleation was performed. Histopathological analysis confirmed the diagnosis of compound odontoma. A literature review was carried out for a deeper discussion of the topic.

Descriptors: Odontogenic Tumors; Odontoma; Tooth Eruption; Diagnosis.

\section{Resumen}

Los odontomas son el tipo más común de tumores odontogénicos benignos. Clínicamente, son asintomáticos y de crecimiento lento, se dividen de acuerdo con las características histomorfológicas en (a) odontomas compuestos, que contienen tejidos dentales normales y están formados por múltiples estructuras calcificadas, algunas parecidas a mini dientes; y (b) odontomas complejos, cuando están presentes como masas irregulares que contienen diferentes tejidos dentales. Por lo general, están asociados con la erupción tardía de los dientes. Un niño de 8 años asistió a la Clínica Odontológica Universitaria con dudas sobre el retraso en la exfoliación de los dientes permanentes. La radiografía periapical mostró la presencia de un odontoma compuesto en la región de los incisivos superiores en el lado izquierdo, lo que constituye un obstáculo para la erupción permanente de los dientes. Bajo anestesia local, se obtuvo acceso al tumor por vía intra oral y se realizó su enucleación quirúrgica. El análisis histopatológico confirmó el diagnóstico de odontoma compuesto. Se realizó una revisión de la literatura para una discusión más profunda del tema.

Descriptores: Tumores Odontogénicos; Odontoma; Erupción Dental; Diagnóstico.

INTRODUÇÃO

Odontomas são tumores calcificados benignos de origem odontogênica, sendo clinicamente considerados como formações tumorais (hamartomas de tecidos dentais) ou anomalias de desenvolvimento, em vez de verdadeiras neoplasias odontogênicas. A etiologia ainda é desconhecida, embora diferentes fatores como traumatismos, infecções ou mutações genéticas possam estar implicados ${ }^{1,2}$.

A incidência de tumores odontogênicos é baixa, variando de $0,002 \%$ a $0,1 \%$, com o odontoma representando a condição benigna mais comum, respondendo por $20 \%$ a $67 \%$ de todos neoplasias odontogênicas ${ }^{3}$. Dois tipos de odontomas, classificados de acordo com a perspectiva histopatológica, são identificados pela Organização Mundial da Saúde (OMS), são eles: odontomas complexos e compostos. Os compostos, em especial, são aqueles nos quais os tecidos dentários são normais, mas seu tamanho e conformação são alterados, dando origem a várias pequenas estruturas semelhantes a dentes, desenvolvendo-se com maior frequência quando comparados com os complexos, não existindo predileção por raça e $\operatorname{sexo}^{4-6}$.

Malformação de dentes adjacentes em desenvolvimento, anodontia, perturbações de erupção e má oclusão são algumas consequências clínicas comuns dessa condição ${ }^{7}$. Entretanto, grande parte das lesões são detectadas durante o exame radiográfico de rotina, em virtude do seu crescimento lento e 
assintomático. A verificação radiográfica e histológica dos odontomas apresentam características peculiares, fundamentais para 0 correto diagnóstico desses tumores ${ }^{8,9}$.

O tratamento comumente empregado para os odontomas, sendo unânime na literatura, é a remoção cirúrgica (exérese) da lesão. A técnica utiliza os mesmos princípios cirúrgicos básicos para extração de dentes inclusos, sendo realizada de forma conservadora $^{10}$.

Nesse sentido, o objetivo deste trabalho é apresentar o caso de um paciente de 9 anos diagnosticado com odontoma composto e oferecer uma revisão da literatura, tendo em vista definir as principais características clínicas e histomorfológicas dessa lesão.

\section{CASO CLÍNICO}

Paciente do sexo masculino, feoderma, 8 anos de idade, compareceu à Clínica Odontopediátrica da Universidade Tiradentes (UNIT) acompanhado da sua responsável, relatando falha na erupção dos dentes anteriores da maxila e inchaço duro na gengiva da região dos incisivos ântero-superiores. Durante a entrevista dialogada, o histórico médico do paciente não mostrou-se contributivo, entretanto um registro de trauma dentário na região anterior foi reportado durante a infância da criança. $O$ exame intra oral revelou que o paciente estava em fase de dentição mista, com ausência dos elementos decíduos 55, 61, 62, 74 e presença dos elementos permanentes 16, 12, $11,26,36$ e 46 . No espaço edêntulo, na região dos incisivos central e lateral superior esquerdo, à palpação a gengiva apresentava-se espessa, fibrosa e com um leve aumento de volume (Figura 1).

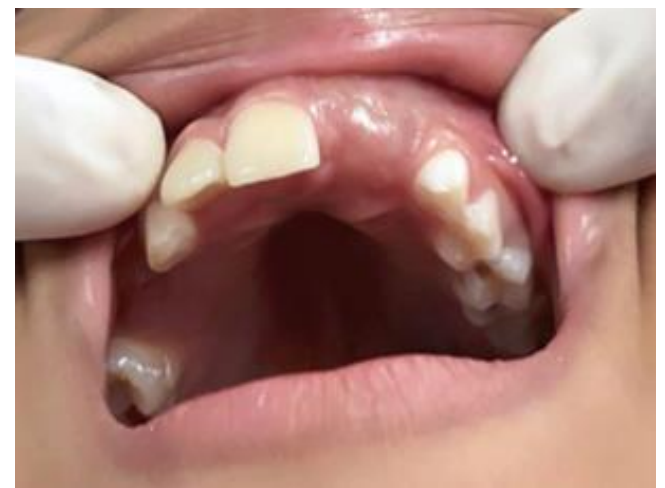

Figura 1: Região endêntula com presença de gengiva espessa, fibrosa e com leve aumento de volume.

O diagnóstico imagionológico foi realizado através de uma radiografia periapical digital da região, onde foi observada uma área radiopaca na região de borda incisal dos incisivos central e lateral superior esquerdo que se encontravam impactados e sobrepostos, sugestivo de odontoma composto. Ambas as unidades estavam em estágio de Nolla avançado e sem espaço na arcada dentária para irrupção (Figura 2). O plano de tratamento proposto baseou-se em biópsia excisional para confirmação do diagnóstico e exodontia da unidade 63, possibilitando livre espaço para ambas as unidades erupcionarem na cavidade bucal.

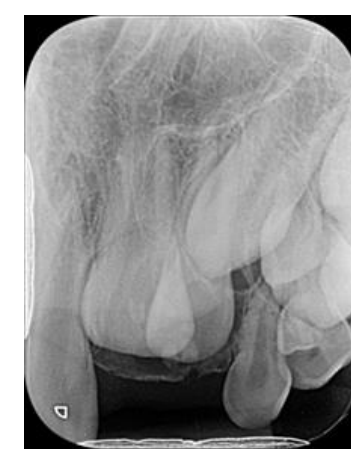

$$
\begin{aligned}
& \text { Figura 2: } \begin{array}{c}
\text { Radiografia } \\
\text { periapical } \\
\text { odontoma }
\end{array} \text { mostrandor o } \\
& \text { impactando a erupção dos } \\
& \text { elementos } 21 \text { e 22, deslocados } \\
& \text { para a região palatina. }
\end{aligned}
$$

A biópsia excisional da lesão foi realizada através do uso da Técnica de Manejo do Reforço Positivo, por se tratar de um paciente infantil e de um procedimento invasivo. A anestesia tópica foi aplicada em fundo de sulco, em seguida anestesia local dos nervos alveolar superior anterior e nervo nasopalatino. Posteriormente, realizou-se a incisão sulcular em volta da unidade 63 , estendendo-se até a mesial da unidade 11; a luxação do dente 62 ocorreu com o uso de uma alavanca reta, já os movimentos de lateralidade e tração com o uso do fórceps número 1 (Figura $3 \mathrm{~A}$ ). Em seguida foi rebatido um retalho envelope até a papila mesial da unidade 11 , realizada a remoção do osso alveolar com o Descolador de Molt $n^{\circ}$ 9, até ser possível a visualização da lesão. Foram removidos 07 fragmentos de tamanhos variados (Figura 3B). A sutura realizada foi em " $x$ " na região de canino, e sutura do tipo simples na região da papila mesial da unidade 11 , deixando a região da unidade 21 e 22, sem sutura na tentativa de ajudar as unidades impactadas a erupcionarem. Foram armazenados 05 fragmentos em um frasco coletor contendo formol e encaminhados ao atendimento especializado em diagnóstico estomatológico. Ao exame macroscópico foi observado que o maior e menor espécime era de 0,2 x 0,2 × 0,2 $\mathrm{cm}$ e 0,1 x 0,1 x 0,1 cm, respectivamente. Apresentavam consistência pétrea, formato ovoide e superfícies lisas, exibindo coloração branca (Figura 3C). No exame microscópico foi observada secções histológicas coradas em HE que revelaram fragmentos de tecido dentinário tubular compacto, além de tecido conjuntivo fibroso hialino moderadamente celularizado e bem vascularizado (Figura 4). A análise 
histopatológica confirmou a hipótese diagnóstica de odontoma composto.

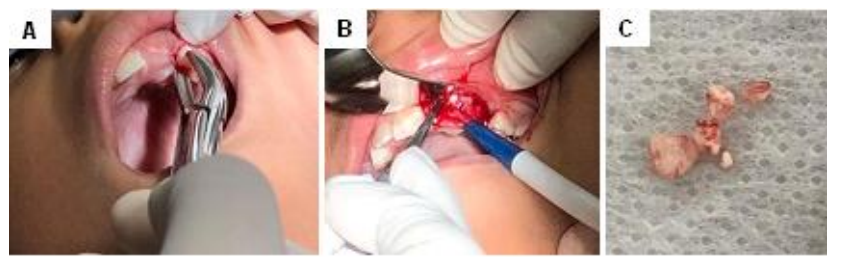

Figura 3: Figura 3A: exodontia do elemento 63 . Figura 3B: biópsia excisional do odontoma. Figura 3C: fragmentos da lesão.
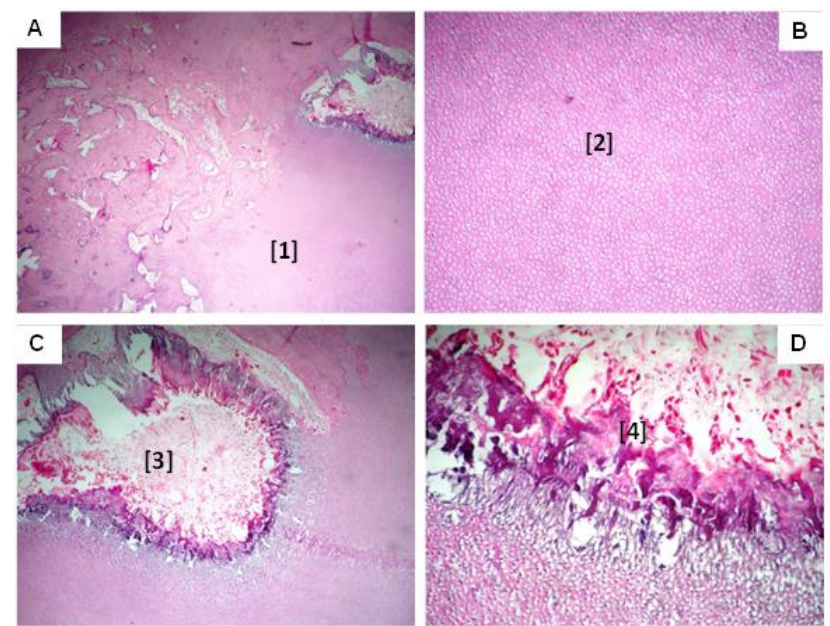

Figura 4: Corte histológico dos fragmentos da lesão corados em $\mathrm{HE}$, apresentando [1] formação compacta de tecido dentinário; [2] dentina tubular mostrando presença de corpo odontoblástico em seu interior; [3] tecido conjuntivo fibroso semelhante à polpa; [4] camada odontoblástica na área da polpa exibindo calcificação distrófica em toda sua extensão.

O paciente foi acompanhado por 11 meses e não haviam indícios dos dentes retidos erupcionarem, sendo assim foi solicitado uma Tomografia Computadorizada de Feixe Cônico (TCFC), a fim de avaliar a possibilidade das unidades dentárias estavam anquilosadas. A TCFC mostrou que a unidade 21 encontra-se inclusa em posição vertical, com rizogênese incompleta, relação com a cortical óssea vestibular e observa-se aumento do folículo pericoronário. A unidade 22 encontra-se inclusa com eixo de inclinação para vestibular, a coroa localizada por palatina em relação a coroa do dente 21 (incluso) e dente 23, apresentando relação de proximidade com as mesmas, a raiz apresentando relação com a cortical óssea palatina e rizogênese incompleta. Unidade 23 encontra-se em formação com alteração no eixo de erupção, a coroa estava localizada por vestibular em relação a coroa do dente 22 , apresentando relação de proximidade com a mesma, observa-se aumento do folículo pericoronário (Figura 5). A TCFC foi avaliada por cirurgião-dentista ortodontista, 0 qual observou que as unidades 21 e 22 não se encontravam anquilosadas, mas, por estarem em estágio de Nolla avançado, não teriam a força eruptiva necessária para irromper espontaneamente na cavidade bucal.
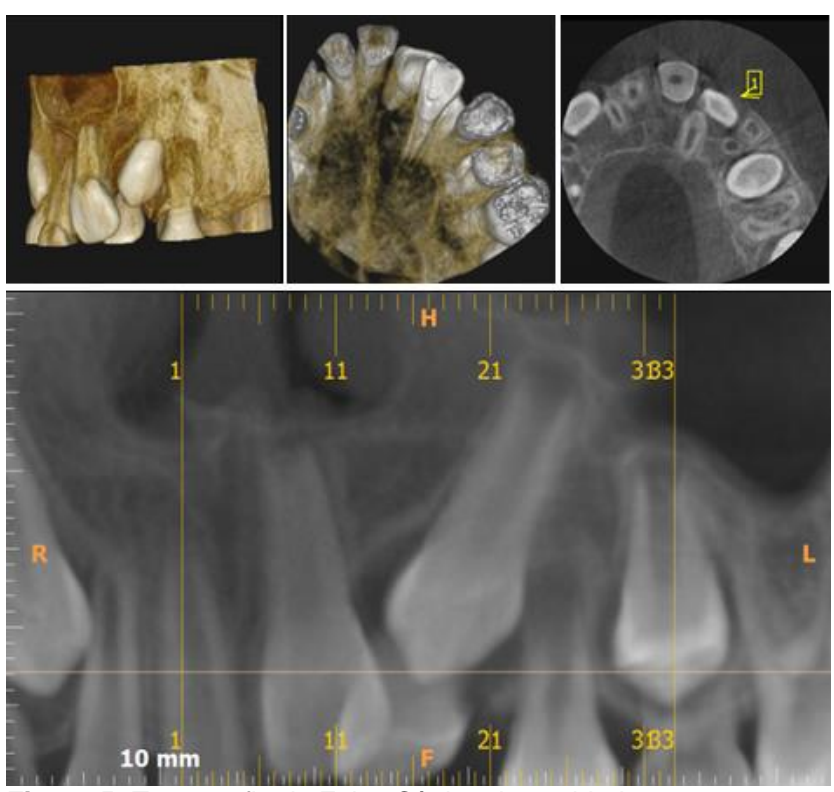

Figura 5: Tomografia de Feixe Cônico das unidades 21, 22 e 23.

O tratamento ideal neste caso seria tracionamento ortodôntico. $O$ paciente foi encaminhado à um ortodontista para conclusão do tratamento.

DISCUSSÃO

Os odontomas são os tumores odontogênicos benignos mais frequentes no campo da patologia oral. A maioria dos casos se mostra assintomática e constitui achado casual no decurso de verificações radiográficas da rotina clínica, acometendo principalmente a segunda e terceira décadas de vida ${ }^{11,12}$. De acordo estudo de meta-analise realizado por Schancez et al. $^{5}$, onde 3065 casos de odontomas foram investigados, em mais da metade desses, algumas manifestações foram predominantemente observadas - inchaço (14\%), persistência dos dentes permanentes em boca $(12,7 \%)$, agenesia dos dentes permanentes $(7,2 \%)$, sendo a condição mais comum a retenção da dentição permanente $(55,4 \%)$. O relato de caso aqui apresentado apresenta caráter semelhante aos dados encontrados na literatura médica, exceto pelo fato na qual o paciente encontrava-se na primeira década de vida. O odontoma foi descoberto através de uma solicitação estética e funcional, sendo responsável pela retenção da dentição permamente do paciente, desenvolvendo-se de forma lenta e assintomática.

Existem dois tipos de odontomas: odontomas complexos e odontomas compostos - sendo o último duas vezes mais frequente que o primeiro ${ }^{13,14}$. De acordo com alguns autores, esses tumores podem ser encontrados em qualquer região dos ossos maxilares, entretanto os odontomas compostos mostram predileção 
pelo setor anterior da maxila superior, enquanto odontomas complexos são tipicamente encontrados na região posterior da mandíbula ${ }^{11}$, 15, 16. Esses achados são comuns aos detalhes clínicos aqui relatados, onde o odontoma composto, mais frequentemente encontrado, foi diagnosticado no paciente, acometendo os incisivos superiores do lado esquerdo, a região de predileção desse tipo tumor.

Segundo Kämmerer et al. ${ }^{17}$ e Pereira et al. ${ }^{18}$, o aumento progressivo do odontoma causa força suficiente capaz de gerar reabsorção radicular dos dentes adjacentes. No caso clínico exposto, as unidades dentárias adjacentes se encontravam em posição normal, sem apresentar reabsorção e sem contato da raiz com a lesão, já que a mesma se encontrava na porção incisal dos incisivos central e lateral superior esquerdo, fazendo com que estes permanecessem impactados.

A etiologia exata desta patologia não foi claramente identificada, mas algumas associações são verificadas aos traumas dentários, origem inflamatória e infecção, histórico familiar e mutação genética, sendo consideradas fatores predisponentes que contribuem para a ocorrência de odontomas ${ }^{17,19}$. Estudos evidenciam que há uma maior prevalência de traumatismo na dentição decídua, quando comparada à permanente, em especial na idade escolar, podendo atingir uma média de $36,8 \%$ das crianças $^{20,21}$. Consistente com o que é relatado na literatura, no presente caso o paciente relatou lembrar que sofreu trauma na dentição decídua enquanto jogava futebol na pré-escola. Essa ocorrência é justificada uma vez que nesta fase, as características comportamentais, como a curiosidade, inquietação e interações sociais levam a criança a explorar o ambiente, e muitas vezes estas não possuem coordenação motora suficiente para evitar quedas e promover autoproteção, o que leva o desenvolvimento desses tipos de lesões ${ }^{22,23}$.

Radiograficamente, os odontomas compostos aparecem como pequenos dentes, confinados em um mesmo espaço, envoltos por halo radiolúcido, enquanto que os odontomas complexos apresentam-se como aglomerados amorfos e radiopacos ${ }^{24}$. As duas variantes apresentam-se na radiografia próximas a dentes, ou entre as raízes ou sobre a coroa de dentes impactados, características peculiares que fazem com que os achados radiográficos sejam diagnósticos, sendo o odontoma composto raramente confundido com outra lesão ${ }^{11}$. No caso apresentado, o diagnóstico foi realizado mediante a realização do exame imagionológico e a análise histopatológica foi processada para verificação da histomorfologia das estruturas presentes no odontoma.

$\mathrm{Na}$ verificação histológica, o odontoma composto apresenta múltiplas estruturas constituídas por esmalte maduro, envoltas em uma matriz fibrosa frouxa, com a presença de tecido pulpar na porção radicular e coronal das estruturas semelhantes a dentes ${ }^{11,25}$. Foram encaminhados ao laboratório 5 dentículos de distintas formas e tamanhos, onde ao exame histopatológico foram observadas características teciduais semelhantes àquelas descritas na literatura.

Os odontomas compostos devem ser tratados por excisão local simples por se tratarem de lesões pequenas e de fácil enucleação ${ }^{10,11,26}$. No presente caso 0 tratamento empregado foi a retirada total da lesão através da biópsia excisional. Embora a literatura relate que a remoção do odontoma é seguida por uma rápida erupção espontânea do dente correspondente impactado ${ }^{17}$, neste trabalho 0 paciente apresentava-se com as unidades inclusas e impactadas, desta forma, orientou-se 0 encaminhamento para 0 tracionamento ortodôntico, devido a resposta eruptiva negativa dos dentes após a remoção da lesão. $O$ fato dos dentes permanentes não erupcionarem pode ter relação com o estágio de Nolla avançado que as unidades dentárias se encontram.

CONCLUSÃO

Com base na literatura e na descrição do caso clínico, nota-se a importância do profissional cirurgião-dentista deter o conhecimento adequado para atender as demandas que 0 tratamento de odontomas exige, uma vez que i) o diagnóstico e a intervenção precoce em casos de retenção prolongada de dentes permanentes são importantes para reduzir ou até mesmo evitar prejuízos à oclusão; ii) a impactação prolongada da dentição permanente pode ser influenciada por fatores locais, ambientais e hereditários, podendo levar ao desenvolvimento de problemas estéticos e funcionais; iii) o tratamento adequado consiste na enucleação do odontoma, seguido da manutenção do espaço, além do controle periódico até a completa erupção da dentição.

\section{REFERÊNCIAS}

1. Ferrer Ramírez MJ, Silvestre Donat FJ, Estelles Ferriol E, Grau García Moreno D, López Martínez R. Recurrent infection of a complex odontoma following eruption in the mouth. Med Oral. $2001 ; 6(4): 269-75$. 
2. Amado Cuesta S, Gargallo Albiol J, Berini Aytés L, Gay Escoda C. Review of 61 cases of odontoma. Presentation of an erupted complex odontoma. Med Oral. 2003;8(5):366-73.

3. Boffano P, Zavattero E, Roccia F, Gallesio C. Complex and compound odontomas. J Craniofac Surg. 2012;23(3):685-88.

4. Ladeinde AL, Ajayi OF, Ogunlewe MO, Adeyemo WL, Arotiba GT, Bamgbose BO, Akinwande JA. Odontogenic tumors: a review of 319 cases in a Nigerian teaching hospital. Oral Surg Oral Med Oral Pathol Oral Radiol Endod. 2005;99(2):191-95.

5. Hidalgo-Sánchez O, Leco-Berrocal MI, Martínez-González JM. Metaanalysis of the epidemiology and clinical manifestations of odontomas. Med Oral Patol Oral Cir Bucal. 2008;13(11):E730-34.

6. Chang JY, Wang JT, Wang YP, Liu BY, Sun A, Chiang CP. Odontoma: a clinicopathologic study of 81 cases. J Formos Med Assoc. 2003;102(12):876-82.

7. Soni V, Buch B, Kotsane D. Radiographic assessment of multiple pathoses and dental malocclusion: a case study. S Afr dent j. 2019;74(3):123-25.

8. Carvalho CHP, Costa DA, Queiroz LMG, Amaral JIQ, Germano AR. Extensive complex odontoma in the maxillary sinus: An uncommon presentation as a cause of chronic sinusitis. Rev odonto ciênc. 2011;26(1):92-5.

9. Torul D, Keskin M, Gun S, Odabasi D. Complex-Compound Odontoma: A Rare Clinical Presentation.Odovtos. 2020;22(1):23-8.

10. Nóia CF, dos Santos Júnior JP, Pinto JMV, Rodrigues MTV. Odontoma CompostoComplexo: relato de caso. RBPS. 2008;10(4):59-63.

11. Neville BW, Damm DD, Allen CM, Bouquot JE. Patologia Oral e Maxilofacial. 3. Ed. Rio de Janeiro: Elsevier; 2009,

12. Weismann $R$, Chaves Júnior $A C$, Panarello AF, Beltrão RG. Odontoma complexo: relato de caso clínico com 12 anos de acompanhamento. Rev ABO Nac. 2007;14(2):105-7.

13. Patiño Illa CP, Berini Aytés L, Sánchez Garcés MA, Gay Escoda C. Odontomas complejos y compuestos: Análisis de 47 casos. Arch odontoestomatol. 1995;11(8):423-30.

14. Philipsen HP, Reichart PA, Praetorius F. Mixed odontogenic tumours and odontomas. Considerations on interrelationship. Review of the literature and presentation of 134 new cases of odontomas. Oral Oncol. 1997;33(2):86-99.

15. Lodi CS, Rodrigues AM, Diana NB, Faria MD, Bordon AKCB, Pereira FP. Odontoma composto em paciente infantil: relato de caso. Arch Health Invest. 2014;3(2):26-33.

16. latrou I, Vardas E, Theologie-Lygidakis N,
Leventis M. A retrospective analysis of the characteristics, treatment and follow-up of 26 odontomas in Greek children. J Oral Sci. 2010;52(3):439-47.

17. Kämmerer PW, Schneider D, Schiegnitz E, Schneider S, Walter C, Frerich B et al. Clinical parameter of odontoma with special emphasis on treatment of impacted teeth-a retrospective multicentre study and literature review. Clin Oral Investig. 2016;20(7):1827-35.

18. Pereira LC, Miceli ALC, Louro RS. Complex odontoma extensive in jaw-Review and Report. Rev cir traumatol buco-maxilo-fac. 2015;15(4):49-52.

19. Arunkumar K, Vijaykumar NG. Surgical management of an erupted complex odontoma occupying maxillary sinus. Ann Maxillofac Surg. 2012;2(1):86-9.

20. Granville-Garcia AF, De Menezes VA, De Lira PIC. Dental trauma and associated factors in Brazilian preschoolers. Dent Traumatol. 2006;22(6):318-22.

21. Schatz J-P, Hausherr C, Joho J-P. A retrospective clinical and radiologic study of teeth re-implanted following traumatic avulsion. Dent Traumatol. 1995;11(5):235-39.

22. da Silva Assunção LR, Cunha RF, Ferelle A. Análise dos traumatismos e suas seqüelas na dentição decídua: uma revisão da literatura. Pesq Bras Odontoped Clín Integr. 2007;7(2):173-79.

23. Vasconcellos R, Oliveira D, Nogueira R, Maciel A, Cordeiro M. Trauma na dentição decídua: enfoque atual. Rev cir traumatol buco-maxilofac. 2003;3(2):17-24.

24. Angiero F, Benedicenti S, Parker S, Signore A, Sorrenti E, Giacometti E, et al. Clinical and surgical management of odontoma. Photomed Laser Surg. 2014;32(1):47-53.

25. Araújo KM. Odontoma dos ossos gnáticos: estudo clinicopatológico de 115 casos. 2014. 22f. Monografia (Graduação) - Departamento de Odontologia, Universidade Federal do Rio Grande do Norte, Natal, 2014.

26. Jaeger $F$, Alvarenga RL, Lage FO, Reis IA, Leal RM. Odontoma composto-relato de caso clínico. Rev Port Estomatol Med Dent Cir Maxilofac. 2012;53(4):252-7. 


\section{CONFLITO DE INTERESSES}

Os autores declaram não haver conflitos de interesse

\section{AUTOR PARA CORRESPONDÊNCIA}

Vanessa dos Santos Viana

Departamento de Odontopediatria

Universidade Tiradentes (UNIT)

Rua Siriri, 263 sala 101,

49010-390 Aracaju/SE Brasil

E-mail: vanessaodontopediatria@gmail.com

Submetido em 17/02/2020

Aceito em 08/03/2021 